Repositorio Institucional de la Universidad Autónoma de Madrid

https://repositorio.uam.es

Esta es la versión de autor del artículo publicado en:

This is an author produced version of a paper published in:

JOURNAL OF SCIENTIFIC COMPUTING 74.1 (2018): 456-473

DOI: $\quad$ https://doi.org/10.1007/s10915-017-0447-2

Copyright: @ 2017 Springer+Business Media New York.

El acceso a la versión del editor puede requerir la suscripción del recurso Access to the published version may require subscription 


\title{
Two-grid mixed finite-element approximations to the Navier-Stokes equations based on a Newton-type step
}

\author{
Francisco Durango Julia Novo*
}

February 21, 2017

\begin{abstract}
A two-grid scheme to approximate the evolutionary Navier-Stokes equations is introduced and analyzed. A standard mixed finite element approximation is first obtained over a coarse mesh of size $H$ at any positive time $T>0$. Then, the approximation is postprocessed by means of solving a steady problem based on one step of a Newton iteration over a finer mesh of size $h<H$. The method increases the rate of convergence of the standard Galerkin method in one unit in terms of $H$ and equals the rate of convergence of the standard Galerkin method over the fine mesh $h$. However, the computational cost is essentially the cost of approaching the Navier-Stokes equations with the plain Galerkin method over the coarse mesh of size $H$ since the cost of solving one single steady problem is negligible compared with the cost of computing the Galerkin approximation over the full time interval $(0, T]$. For the analysis we take into account the loss of regularity at initial time of the solution of the Navier-Stokes equations in the absence of nonlocal compatibility conditions. Some numerical experiments are shown.
\end{abstract}

Keywords Incompressible Navier-Stokes equations; inf-sup stable finite element methods; static two-grid methods; nonlocal compatibility conditions

\section{Introduction}

Let $\Omega$ be a bounded domain in $\mathbb{R}^{d}(d=2,3)$ with a smooth boundary $\partial \Omega$ and let us consider the incompressible Navier-Stokes equations

$$
\begin{array}{r}
u_{t}-\nu \Delta u+(u \cdot \nabla) u+\nabla p=f \\
\operatorname{div}(u)=0
\end{array}
$$

*Departamento de Matemáticas, Universidad Autónoma de Madrid, Spain. Research supported by Spanish MEC under grant MTM2013-42538-P (julia.novo@uam.es) 
with initial condition $u(\cdot, 0)=u_{0}$ and homogeneous Dirichlet conditions $u=0$ on the boundary. In this paper, we study a two-grid postprocessing technique based on Newton iteration. First, a mixed finite-element approximation $\left(u_{H}, p_{H}\right)$ is computed over a coarse grid of size $H$. Then, the postprocessed solution $(\tilde{u}, \tilde{p})$ is obtained at any time $T>0$ as the mixed finite element approximation over a fine mesh $h<H$ to the following steady problem:

$$
\begin{array}{r}
-\nu \Delta \tilde{u}+\left(u_{H} \cdot \nabla\right) \tilde{u}+(\tilde{u} \cdot \nabla) u_{H}+\nabla \tilde{p}+\lambda \tilde{u}=f-\frac{d}{d t} u_{H}+\left(u_{H} \cdot \nabla\right) u_{H}+\lambda u_{H} \\
\operatorname{div}(\tilde{u})=0
\end{array}
$$

where $\lambda$ is a positive parameter that is added to have coercivity in the bilinear form associated to problem (2) whose value will be specified later.

In general, two-grid postprocessing techniques improve the rate of convergence of the standard finite element approximations with a slight increase in the computational cost. In [24], [23] several two-level methods are considered to approximate the steady Navier-Stokes equations. They require solving a nonlinear system over a coarse mesh and, depending on the algorithm chosen, one Stokes problem, one linear Oseen problem or one Newton step over the fine mesh. The corresponding algorithms obtain the optimal rate of convergence in the fine mesh for appropriate choices of the coarse mesh diameter $\mathrm{H}$.

Static two-grid or two-grid postprocessing techniques for the evolutionary Navier-Stokes equations have also been studying. In all cases the plain Galerkin method is used to evolve in time and a steady problem is solved at the fine level. More precisely, the problem solved at the fixed step or fine level can be a Stokes problem or an Oseen-type problem both computed with data based on the Galerkin approximation. The Stokes-type postprocess was first developed for spectral methods in [6], [7], [13], [26] and then applied to mixed finite element methods in [2], [3], [11], [12]. The Oseen-postprocess has been studied in [15]. In both cases, the rate of convergence of the postprocessed approximation equals the rate or convergence of the standard finite element approximation over the fine grid and improves in one unit the rate of convergence of the Galerkin method over the coarse grid.

Several authors have also considered two-grid algorithms for the evolutionary Navier-Stokes equations based on evolutionary linearized problems. We refer the reader to [16] for a detailed description of several algorithms of this type that have appeared in the literature. We add to those references two more that have recently appeared. In [19] a two level method based on a linearized time dependent Stokes problem is analyzed considering the case of non-smooth initial data. In [25] Euler-based discretizations of three corrections based on linearized time dependent Stokes, Oseen and Newton problems are considered.

In this paper we concentrate ourselves on the static two-grid approach based on a Newton-type step to the evolutionary Navier-Stokes equations. The reason is twofold, on the one hand the static approaches are computationally cheaper than the dynamical approaches and on the other, although there are three typical linearized methods that can be applied: Stokes, Oseen and Newton, to our 
knowledge, the last one had not been previously analyzed. We show that with the static two grid method based on Newton-type postprocess we can improve the rate of convergence of the Galerkin approximation in one unit. The inclusion of the reaction term $(\tilde{u} \cdot \nabla) u_{H}$, with the subsequent lost of coercivity of the bilinear form associated to the steady problem, introduces several challenges which are of interest.

As in [11], [12], [15] for the analysis we take into account the loss of regularity suffered by the solutions of the Navier-Stokes equations at the initial time in the absence of nonlocal compatibility conditions. Consequently, for the analysis we do not assume the solution $u$ to have more than second-order partial derivatives bounded in $L^{2}$ up to the initial time $t=0$. Demanding further regularity requires the data to satisfy nonlocal compatibility conditions unlikely to be fulfilled in practical situations, see [20], [21]. Due to the loss of regularity at $t=0$, the best error bound that we can obtain is $O\left(H^{5}|\log (H)|\right)$. For this reason we do not analyze higher than cubic finite elements. The same limit in the rate of convergence was found in [21] for standard mixed finite-element approximations and in [11], [15], [16] for two-grid schemes.

The outline of the paper is as follows. We first introduce some preliminaries and notations. In Section 3 we describe the three static linearized approaches to the nonlinear Navier-Stokes equations. The new method is analyzed in Section 5 , based on some theoretical results obtained in Section 4. Finally, last section is devoted to show some numerical experiments. We check numerically the rate of convergence of the method predicted by the theory and we compare the static Newton-type approach with the static Stokes and Oseen approaches for different values of the diffusion parameter in order to study the behaviour of the different methods for different values of the Reynolds number.

\section{Preliminaries and notations}

Along the paper we will denote by $W^{m, q}(\Omega)^{d}$ the space of Lebesgue integrable functions with $m$ (weak) derivatives in $L^{q}(\Omega)$. For $q=2$ we will use the standard notation $H^{m}(\Omega)^{d}=W^{m, 2}(\Omega)^{d}$.

For $q \in[1, \infty)$, we will use the following Sobolev embedding formula. There exists a constant $C$ depending on the domain such that

$$
\|v\|_{L^{q^{\prime}}} \leq C\|v\|_{W^{s, q}}, \quad \frac{1}{q^{\prime}} \geq \frac{1}{q}-\frac{s}{d}>0, \quad q<\infty, \quad v \in W^{s, q}(\Omega)^{d} .
$$

For $q^{\prime}=\infty$, (3) holds with $\frac{1}{q}<\frac{s}{d}$. In particular, we will do extensive use of the following cases, which hold simultaneously for both two and three spatial dimensions.

$$
\|v\|_{L^{2 d}} \leq C\|v\|_{s}, \quad s \geq 1, \quad\|v\|_{L^{2 d /(d-1)}} \leq C\|v\|_{s}, \quad s \geq 1 / 2 .
$$

We consider the Hilbert spaces:

$$
\begin{aligned}
\mathcal{H} & =\left\{u \in L^{2}(\Omega)^{d} \mid \operatorname{div}(u)=0, u \cdot n_{\mid \partial \Omega}=0\right\}, \\
V & =\left\{u \in H_{0}^{1}(\Omega)^{d} \mid \operatorname{div}(u)=0\right\},
\end{aligned}
$$


endowed respectively with the $L^{2}(\Omega)^{d}$ and $H^{1}(\Omega)^{d}$ norms. The following inf-sup condition (see [18]) is satisfied

$$
\inf _{q \in L^{2}(\Omega) / \mathbb{R}} \sup _{v \in H_{0}^{1}(\Omega)^{d}} \frac{(q, \nabla \cdot v)}{\|q\|_{L^{2}(\Omega) / \mathbb{R}}\|v\|_{1}} \geq \beta \geq 0 .
$$

For $u \in V, v, w \in H_{0}^{1}(\Omega)^{d}$, it also holds

$$
((u \cdot \nabla) v, w)=-((u \cdot \nabla) w, v)=-(\nabla \bar{w} \cdot v, u)
$$

where $(\nabla \bar{w})_{i j}=\partial_{i} w_{j}$.

Let $\Pi: L^{2}(\Omega)^{d} \longrightarrow \mathcal{H}$ be the $L^{2}(\Omega)^{d}$ projection onto $\mathcal{H}$. We denote by $A$ the Stokes operator on $\Omega$ :

$$
A: \mathcal{D}(A) \subset \mathcal{H} \longrightarrow \mathcal{H}, \quad A=-\Pi \Delta, \quad \mathcal{D}(A)=H^{2}(\Omega)^{d} \cap \mathcal{V} .
$$

We shall assume that $u$ is a strong solution up to time $t=T$, so that

$$
\|u(t)\|_{1} \leq M_{1}, \quad\|u(t)\|_{2} \leq M_{2}, \quad 0 \leq t \leq T,
$$

for some constants $M_{1}$ and $M_{2}$. We shall also assume that there exists a constant $\tilde{M}_{2}$ such that

$$
\|f\|_{1}+\left\|f_{t}\right\|_{1}+\left\|f_{t t}\right\|_{1} \leq \tilde{M}_{2}, \quad 0 \leq t \leq T .
$$

Finally, we shall assume that for some $k \geq 2$

$$
\sup _{0 \leq t \leq T}\left\|\partial_{t}^{\lfloor k / 2\rfloor} f\right\|_{k-1-2\lfloor k / 2\rfloor}+\sum_{j=0}^{\lfloor(k-2) / 2\rfloor} \sup _{0 \leq t \leq T}\left\|\partial_{t}^{j} f\right\|_{k-2 j-2}<+\infty,
$$

so that, according to Theorems 2.4 and 2.5 in [20], there exist positive constants $M_{k}$ and $K_{k}$ such that the following bounds hold:

$$
\begin{gathered}
\|u(t)\|_{k}+\left\|u_{t}(t)\right\|_{k-2}+\|p(t)\|_{H^{k-1} / \mathbb{R}} \leq M_{k} \tau(t)^{1-k / 2}, \\
\int_{0}^{t} \sigma_{k-3}(s)\left(\|u(s)\|_{k}^{2}+\left\|u_{s}(s)\right\|_{k-2}^{2}+\|p(s)\|_{H^{k-1} / \mathbb{R}}^{2}+\left\|p_{s}(s)\right\|_{H^{k-3} / \mathbb{R}}^{2}\right) \mathrm{d} s \leq K_{k}^{2},
\end{gathered}
$$

where $\tau(t)=\min (t, 1)$ and $\sigma_{n}=e^{-\alpha(t-s)} \tau^{n}(s)$ for some $\alpha>0$. Observe that for $t \leq T<\infty$, we can take $\tau(t)=t$ and $\sigma_{n}(s)=s^{n}$. For simplicity, we will take these values of $\tau$ and $\sigma_{n}$. We want to remark that in the error bounds we prove in this paper the final time $T$ is fixed and we are not studying the behavior of the bounds for increasing values of $T$ while, on the contrary, we want to clarify the behavior of the bounds around $t=0$.

Let $\mathcal{T}_{h}=\left(\tau_{i}^{h}, \phi_{i}^{h}\right)_{i \in I_{h}}, h>0$ be a family of partitions of suitable domains $\Omega_{h}$, where $h$ is the maximum diameter of the elements $\tau_{i}^{h} \in \mathcal{T}_{h}$, and $\phi_{i}^{h}$ are the mappings of the reference simplex $\tau_{0}$ onto $\tau_{i}^{h}$. Let $r \geq 2$, we consider the finite-element spaces

$$
\begin{aligned}
S_{h, r} & =\left\{\chi_{h} \in \mathcal{C}\left(\bar{\Omega}_{h}\right)\left|\chi_{h}\right|_{\tau_{i}^{h}} \circ \phi_{i}^{h} \in P^{r-1}\left(\tau_{0}\right)\right\} \subset H^{1}\left(\Omega_{h}\right), \\
S_{h, r}^{0} & =S_{h, r} \cap H_{0}^{1}\left(\Omega_{h}\right),
\end{aligned}
$$


where $P^{r-1}\left(\tau_{0}\right)$ denotes the space of polynomials of degree at most $r-1$ on $\tau_{0}$. We shall denote by $\left(X_{h, r}, Q_{h, r-1}\right)$ the so-called Hood-Taylor element [4, 22], when $r \geq 3$, where

$$
X_{h, r}=\left(S_{h, r}^{0}\right)^{d}, \quad Q_{h, r-1}=S_{h, r-1} \cap L^{2}\left(\Omega_{h}\right) / \mathbb{R}, \quad r \geq 3,
$$

and the so-called mini-element [8] when $r=2$, where $Q_{h, 1}=S_{h, 2} \cap L^{2}\left(\Omega_{h}\right) / \mathbb{R}$, and $X_{h, 2}=\left(S_{h, 2}^{0}\right)^{d} \oplus \mathbb{B}_{h}$. Here, $\mathbb{B}_{h}$ is spanned by the bubble functions $b_{\tau}, \tau \in \mathcal{T}_{h}$, defined by $b_{\tau}(x)=(d+1)^{d+1} \lambda_{1}(x) \cdots \lambda_{d+1}(x)$, if $x \in \tau$ and 0 elsewhere, where $\lambda_{1}(x), \ldots, \lambda_{d+1}(x)$ denote the barycentric coordinates of $x$. For these elements a uniform inf-sup condition is satisfied (see [4]), that is, there exists a constant $\beta>0$ independent of the mesh grid size $h$ such that

$$
\inf _{q_{h} \in Q_{h, r-1}} \sup _{v_{h} \in X_{h, r}} \frac{\left(q_{h}, \nabla \cdot v_{h}\right)}{\left\|v_{h}\right\|_{1}\left\|q_{h}\right\|_{L^{2} / \mathbb{R}}} \geq \beta .
$$

The approximate velocity belongs to the discrete divergence-free space

$$
V_{h, r}=X_{h, r} \cap\left\{\chi_{h} \in H_{0}^{1}\left(\Omega_{h}\right)^{d} \mid\left(q_{h}, \nabla \cdot \chi_{h}\right)=0 \quad \forall q_{h} \in Q_{h, r-1}\right\} .
$$

Let $\left(u_{H}, p_{H}\right) \in\left(X_{H, r}, Q_{H, r-1}\right)$ be the semi discrete Galerkin approximation to the exact solution $(u, p)$ of the Navier-Stokes equations, that is for $t \in(0, T]$, $\left(u_{H}, p_{H}\right)$ is the solution of the following problem for all $\phi_{H} \in X_{H, r}$ and $\psi_{H} \in$ $Q_{H, r-1}$

$$
\begin{aligned}
\left(\dot{u}_{H}, \phi_{H}\right)+\nu\left(\nabla u_{H}, \nabla \phi_{H}\right)+b\left(u_{H}, u_{H}, \phi_{H}\right)+\left(\nabla p_{H}, \phi_{H}\right) & =\left(f, \phi_{H}\right), \\
\left(\nabla \cdot u_{H}, \psi_{H}\right) & =0,
\end{aligned}
$$

where $b(u, v, w)=\left((u \cdot \nabla) v+\frac{1}{2}(\nabla \cdot u) v, w\right)$ for any $u, v, w \in H_{0}^{1}(\Omega)^{d}$. The following bounds hold for $2 \leq r \leq 5$, see [11], [20] and [21]

$$
\left\|u(t)-u_{H}(t)\right\|_{0}+H\left\|u(t)-u_{H}(t)\right\|_{1} \leq C \frac{H^{r}}{t^{(r-2) / 2}}, \quad 0 \leq t \leq T,
$$

and also,

$$
\left\|p(t)-p_{H}(t)\right\|_{L^{2} / \mathbb{R}} \leq C \frac{H^{r-1}}{t^{\left(r^{\prime}-2\right) / 2}}, \quad 0 \leq t \leq T,
$$

where $r^{\prime}=r$ if $r \leq 4$ and $r^{\prime}=r+1$ if $r=5$.

\section{The Newton-type problem}

Since Navier-Stokes equations are non-linear, it is of interest to study linearized problems related to them. Stokes, Oseen and Newton-type problems provide three ways to linearize the equations. Let $g \in L^{2}(\Omega)$ and consider the Stokes problem

$$
\left.\begin{array}{rll}
-\nu \Delta v+\nabla j= & g \\
\operatorname{div}(v)= & 0
\end{array}\right\} \quad \begin{array}{ll}
\text { in } \Omega \\
v=0, & \text { on } \partial \Omega
\end{array}
$$


with homogeneous Dirichlet boundary conditions. It is well known (see [21]) that the Stokes projection $\left(s_{h}, q_{h}\right) \in\left(X_{h, r}, Q_{h, r-1}\right)$ that approximates problem (15) satisfies the following error bounds for $2 \leq m \leq r$

$$
\begin{aligned}
\left\|v-s_{h}\right\|_{0}+h\left\|v-s_{h}\right\|_{1} & \leq C h^{m}\left(\|v\|_{m}+\|j\|_{H^{m-1} / \mathbb{R}}\right) \\
\left\|j-q_{h}\right\|_{L^{2} / \mathbb{R}} & \leq C h^{m-1}\left(\|v\|_{m}+\|j\|_{H^{m-1} / \mathbb{R}}\right) .
\end{aligned}
$$

Let us now consider the following steady Oseen-type problem, which can be obtained by adding a convection term to the Stokes problem $(u \cdot \nabla) v$, where $u$ is a function satisfying $\nabla \cdot u=0$.

$$
\left.\begin{array}{rll}
-\nu \Delta v+(u \cdot \nabla) v+\nabla j & =g \\
\operatorname{div}(v) & =0
\end{array}\right\} \quad \begin{array}{ll}
\text { in } \Omega \\
v=0 . & \text { on } \partial \Omega
\end{array}
$$

The mixed finite-element approximation $\left(w_{h}, k_{h}\right) \in\left(X_{h, r}, Q_{h, r-1}\right)$ of the Oseen problem (18) satisfies the same bounds as the Stokes projection [15] for $2 \leq$ $m \leq r$

$$
\begin{aligned}
\left\|v-w_{h}\right\|_{0}+h\left\|v-w_{h}\right\|_{1} & \leq C h^{m}\left(\|v\|_{m}+\|j\|_{H^{m-1} / \mathbb{R}}\right) \\
\left\|j-k_{h}\right\|_{L^{2} / \mathbb{R}} & \leq C h^{m-1}\left(\|v\|_{m}+\|j\|_{H^{m-1} / \mathbb{R}}\right) .
\end{aligned}
$$

Now, we add to the Oseen problem two new terms. The first one is an extra reaction term $(v \cdot \nabla) u$ which transforms the Oseen problem into a Newton-type problem. Since in general the resulting problem is not coercive, we also add a correction term $\lambda v$ to ensure coercivity. The Newton problem we consider along the paper is then the following:

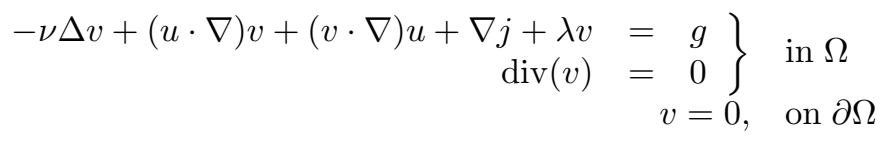

Let us denote by $B_{u}$ the bilinear form associated to problem (21)

$$
B_{u}(v, w)=\nu(\nabla v, \nabla w)+((u \cdot \nabla) v, w)+((v \cdot \nabla) u, w)+\lambda(v, w), \quad v, w \in H_{0}^{1}
$$

Continuity of the bilinear form (22) can be derived from

$$
\begin{aligned}
\left|B_{u}(v, w)\right| \leq & \nu\|v\|_{1}\|w\|_{1}+\|u\|_{L^{2 d /(d-1)}}\|\nabla v\|_{0}\|w\|_{L^{2 d}} \\
& +\|v\|_{L^{2 d /(d-1)}}\|\nabla u\|_{0}\|w\|_{L^{2 d}}+\lambda\|v\|_{0}\|w\|_{0} \\
\leq & \left(\nu+2 C\|u\|_{1}+\lambda\right)\|v\|_{1}\|w\|_{1},
\end{aligned}
$$

where we have applied Sobolev inequality (4).

Coercivity comes from the antisymmetry of the convection term $((u \cdot \nabla) v, v)=$ 0 (6) and the following bound

$$
|((v \cdot \nabla) u, v)| \leq\|\nabla u\|_{\infty}\|v\|_{0}^{2}
$$


that is

$$
B_{u}(v, v) \geq \nu\|\nabla v\|_{0}^{2}+\left(\lambda-\|\nabla u\|_{\infty}\right)\|v\|_{0}^{2} .
$$

Observe that due to the reaction term $((v \cdot \nabla) u, v)$, Newton-type problem becomes non coercive, so it is necessary to include the correction term $\lambda v$ in (21). Now, assuming

$$
\lambda-\|\nabla u\|_{\infty} \geq 0
$$

we get that $B_{u}(v, w)$ is coercive being $\nu$ the constant of coercivity.

Let us observe that the norm $\|\nabla u\|_{\infty}$ is bounded. By (3) and (8) we get

$$
\|\nabla u\|_{\infty} \leq C\|\nabla u\|_{3 / 2+\alpha} \leq C\|u\|_{5 / 2+\alpha} \leq M_{3} t^{-1 / 2}
$$

Since we solve the Newton-type problem at a fixed time $T>0$ we can bound $\|\nabla u\|_{\infty} \leq C M_{3} T^{-1 / 2}$. Let us also observe that since $\|\nabla u\|_{\infty}$ is not known in practice we can replace in $(26)\|\nabla u\|_{\infty}$ by $\left\|\nabla u_{H}\right\|_{\infty}$ to have a computable algorithm.

Remark 1 We want to remark that that assumption $u \in L^{\infty}\left(0, T ; W^{1, \infty}(\Omega)\right)$ is also required in other related references. See for example [5] where the analysis of the continuous interior penalty finite element method for the time-dependent Navier-Stokes equation is considered, [1] where the local projection finite element stabilization for the time-dependent incompressible Navier-Stokes problem is analyzed and [9] where the authors prove error bounds for stabilized finite element methods for the Oberbeck-Boussinesq model.

The bilinear form $B_{u}(v, w)$ is thus continuous and coercive on the whole space $H_{0}^{1}$. In particular, this is also true for the divergence free space $V$. Then, by the Lax-Milgram theorem and the aid of the continuous inf-sup condition (5), there exists a unique solution $(v, j)$ of the problem (21). Regularity conditions can be obtained from the general theory of elliptic problems [17]:

$$
\|v\|_{2}+\|j\|_{H^{1}(\Omega) / \mathbb{R}} \leq C\|g\|_{0} .
$$

Now, by (6), the dual problem of $(21)$ is

$$
\left.\begin{array}{rl}
-\nu \Delta v-(u \cdot \nabla) v-\nabla \bar{v} \cdot u+\lambda v+\nabla j= & g, \\
\operatorname{div}(v)= & 0,
\end{array}\right\} \quad \begin{aligned}
& \text { in } \Omega \\
& v=0, \quad \text { on } \partial \Omega
\end{aligned}
$$

Let $D_{u}(v, w)$ be the bilinear form associated to the problem (27)

$$
D_{u}(v, w)=\nu(\nabla v, \nabla w)-((u \cdot \nabla) v, w)-(\nabla \bar{v} \cdot u, w)+\lambda(v, w)
$$

that satisfies $D_{u}(v, w)=B_{u}(w, v)$. The bilinear form $D_{u}$ is also continuous and coercive so existence, uniqueness and regularity conditions of problem (27) can be obtained arguing exactly as before. In particular the following regularity condition for the dual problem holds true:

$$
\|v\|_{2}+\|j\|_{H^{1}(\Omega) / \mathbb{R}} \leq C\|g\|_{0} .
$$

In the following lemma we get error bounds for the mixed finite-element approximation to problem (21) analogous to bounds (16)-(17) and (19)-(20) for the Stokes (15) and the Oseen (18) problems respectively. 
Lemma 1 Let $u$ be the velocity of the Navier-Stokes equations (1) and let $(v, j)$ be the solution of the linearized problem (21). Consider the discrete variational problem for all $\phi_{h} \in X_{h, r}$ and $\psi_{h} \in Q_{h, r-1}$

$$
\begin{aligned}
\nu\left(\nabla v_{h}, \nabla \phi_{h}\right)+\left((u \cdot \nabla) v_{h}, \phi_{h}\right)+\left(\left(v_{h} \cdot \nabla\right) u, \phi_{h}\right) & +\lambda\left(v_{h}, \phi_{h}\right) \\
+\left(\nabla j_{h}, \phi_{h}\right) & =\left(g, \phi_{h}\right), \\
\left(\nabla \cdot v_{h}, \psi_{h}\right) & =0 .
\end{aligned}
$$

Then, there exists a unique solution $\left(v_{h}, j_{h}\right) \in\left(X_{h, r}, Q_{h, r-1}\right)$ of (29) which satisfies the following bounds for $2 \leq m \leq r$

$$
\begin{aligned}
\left\|v-v_{h}\right\|_{0}+h\left\|v-v_{h}\right\|_{1} & \leq C h^{m}\left(\|v\|_{m}+\|j\|_{H^{m-1} / \mathbb{R}}\right), \\
\left\|j-j_{h}\right\|_{L^{2} / \mathbb{R}} & \leq C h^{m-1}\left(\|v\|_{m}+\|j\|_{H^{m-1} / \mathbb{R}}\right) .
\end{aligned}
$$

Proof Let us observe that the bilinear form $B_{u}(v, w)$ is also coercive and continuous in the discrete divergence free subspace $V_{h, r}$. Applying Lax-Milgram Theorem and the discrete inf-sup condition (10), the existence and uniqueness of the discrete solution of $(29)$ is obtained.

Now, we observe that problem (21) can be rewritten as an Oseen-type problem with right-hand side $g-v \cdot \nabla u-\lambda v$ as follows

$$
\begin{aligned}
-\nu \Delta v+(u \cdot \nabla) v+\nabla j & =g-v \cdot \nabla u-\lambda v, \\
\operatorname{div}(v) & =0 .
\end{aligned}
$$

Let $\left(w_{h}, k_{h}\right) \in\left(X_{h, r}, Q_{h, r-1}\right)$ be the finite-element approximation of (32) defined for all $\left(\phi_{h}, \psi_{h}\right) \in\left(X_{h, r}, Q_{h, r-1}\right)$ by

$$
\begin{aligned}
\nu\left(\nabla w_{h}, \nabla \phi_{h}\right)+\left((u \cdot \nabla) w_{h}, \phi_{h}\right)+\left(\nabla k_{h}, \phi_{h}\right) & =\left(g, \phi_{h}\right)-\left((v \cdot \nabla) u, \phi_{h}\right)-\lambda\left(v, \phi_{h}\right) \\
\left(\nabla \cdot w_{h}, \psi\right) & =0 .
\end{aligned}
$$

We decompose the error in two parts

$$
v-v_{h}=\left(v-w_{h}\right)+\left(w_{h}-v_{h}\right) .
$$

The first term on the right-hand side above is bounded in (19). For the second term $e_{h}=w_{h}-v_{h}$ we subtract (29) from (33) and project onto the divergence free space to get

$$
\begin{gathered}
\nu\left(\nabla e_{h}, \nabla \phi_{h}\right)+\left((u \cdot \nabla) e_{h}, \phi_{h}\right)+\left(\left(e_{h} \cdot \nabla\right) u, \phi_{h}\right)+\lambda\left(e_{h}, \phi_{h}\right) \\
=\left(\left(\left(w_{h}-v\right) \cdot \nabla\right) u, \phi_{h}\right)+\lambda\left(w_{h}-v, \phi_{h}\right), \quad \forall \phi_{h} \in V_{h, r} .
\end{gathered}
$$

Taking $\phi_{h}=e_{h}$ and using the coercivity of the bilinear form $B_{u}$ we get a first estimation of $\left\|e_{h}\right\|_{1}$

$$
\nu\left\|e_{h}\right\|_{1}^{2} \leq\left\|w_{h}-v\right\|_{0}\|\nabla u\|_{\infty}\left\|e_{h}\right\|_{0}+\lambda\left\|w_{h}-v\right\|_{0}\left\|e_{h}\right\|_{0},
$$

from which

$$
\left\|e_{h}\right\|_{1} \leq C\left\|w_{h}-v\right\|_{0} \leq h^{m}\left(\|v\|_{m}+\|j\|_{H^{m-1} / \mathbb{R}}\right),
$$


where in the last inequality we have applied (19).

Inserting (37) in (34) and applying (19) again we complete the proof of the bound for the $H^{1}$ norm in (30)

$$
\left\|v-v_{h}\right\|_{1} \leq C h^{m-1}\left(\|v\|_{m}+\|j\|_{H^{m-1} / \mathbb{R}}\right) .
$$

In view of (37) we have proved a super-convergent result in the $H^{1}$ norm between the Galerkin approximation to the velocity of problem (21) and the Oseen approximation to the velocity of the same problem defined in (33).

For the pressure bound, we decompose

$$
j-j_{h}=\left(j-k_{h}\right)+\left(k_{h}-j_{h}\right)
$$

and subtract again (29) from (33) to obtain

$$
\begin{array}{r}
\nu\left(\nabla e_{h}, \nabla \phi_{h}\right)+\left((u \cdot \nabla) e_{h}, \phi_{h}\right)+\left(\nabla\left(k_{h}-j_{h}\right), \phi_{h}\right)=\left(\left(\left(v_{h}-v\right) \cdot \nabla\right) u, \phi_{h}\right) \\
+\lambda\left(v_{h}-v, \phi_{h}\right) \quad \forall \phi_{h} \in X_{h, r}
\end{array}
$$

Applying the inf-sup condition (10) and (4) it follows

$$
\beta\left\|k_{h}-j_{h}\right\|_{L^{2} / \mathbb{R}} \leq \nu\left\|e_{h}\right\|_{1}+C\|u\|_{1 / 2}\left\|e_{h}\right\|_{1}+C\|u\|_{1}\left\|v_{h}-v\right\|_{1}+\lambda\left\|v_{h}-v\right\|_{1},
$$

so that applying (37) and (38) the proof of (31) is finished.

To bound the zero norm we use duality arguments. Let us observe that

$$
\left\|e_{h}\right\|_{0}=\sup _{\varphi \in L^{2}(\Omega)^{d}} \frac{\left|\left(e_{h}, \varphi\right)\right|}{\|\varphi\|_{0}}
$$

so that for each fixed $\varphi \in L^{2}(\Omega)^{d}$, we introduce the dual problem of (21).

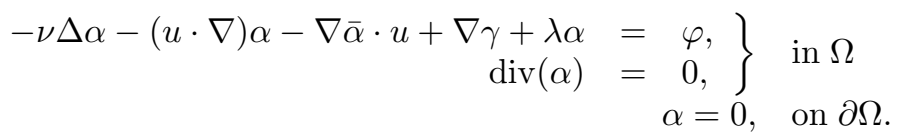

Let $\left(\alpha_{h}, \gamma_{h}\right)$ be the Stokes projection of the solution $(\alpha, \gamma)$ of (40). This approximation satisfies, by (16), (17) and (28), the following bounds

$$
\begin{aligned}
\left\|\alpha-\alpha_{h}\right\|_{0}+h\left\|\alpha-\alpha_{h}\right\|_{1} & \leq C h^{2}\left(\|\alpha\|_{2}+\|\gamma\|_{H^{1} / \mathbb{R}}\right) \leq C h^{2}\|\varphi\|_{0}, \\
\left\|\gamma-\gamma_{h}\right\|_{L^{2} / \mathbb{R}} & \leq C h\left(\|\alpha\|_{2}+\|\gamma\|_{H^{1} / \mathbb{R}}\right) \leq C h\|\varphi\|_{0} .
\end{aligned}
$$

Integrating by parts, we get

$$
\begin{aligned}
\left(\varphi, e_{h}\right)= & \nu\left(\nabla e_{h}, \nabla \alpha\right)+\left((u \cdot \nabla) e_{h}, \alpha\right)+\left(\left(e_{h} \cdot \nabla\right) u, \alpha\right)+\lambda\left(\alpha, e_{h}\right)-\left(\gamma, \nabla \cdot e_{h}\right) \\
= & \nu\left(\nabla e_{h}, \nabla\left(\alpha-\alpha_{h}\right)\right)+\left((u \cdot \nabla) e_{h}, \alpha-\alpha_{h}\right)+\left(\left(e_{h} \cdot \nabla\right) u, \alpha-\alpha_{h}\right) \quad(42) \\
& +\lambda\left(e_{h}, \alpha-\alpha_{h}\right)+\left(\gamma_{h}-\gamma, \nabla \cdot e_{h}\right)+\nu\left(\nabla e_{h}, \nabla \alpha_{h}\right)+\left((u \cdot \nabla) e_{h}, \alpha_{h}\right) \\
& +\left((u \cdot \nabla) e_{h}, \alpha_{h}\right)+\lambda\left(e_{h}, \alpha_{h}\right) .
\end{aligned}
$$


Now the terms depending on $\alpha-\alpha_{h}$ are easily bounded applying (4) and (41)

$$
\begin{aligned}
\left(\varphi, e_{h}\right) \leq & \nu\left\|e_{h}\right\|_{1}\left\|\alpha-\alpha_{h}\right\|_{1}+\|u\|_{1 / 2}\left\|e_{h}\right\|_{1}\left\|\alpha-\alpha_{h}\right\|_{1} \\
& +\|u\|_{1}\left\|e_{h}\right\|_{1}\left\|\alpha-\alpha_{h}\right\|_{1}+\lambda\left\|e_{h}\right\|_{1}\left\|\alpha-\alpha_{h}\right\|_{1}+\left\|\gamma-\gamma_{h}\right\|_{L^{2} / \mathbb{R}}\left\|e_{h}\right\|_{1} \\
& +\nu\left(\nabla e_{h}, \nabla \alpha_{h}\right)+\left((u \cdot \nabla) e_{h}, \alpha_{h}\right)+\left(\left(e_{h} \cdot \nabla\right) u, \alpha_{h}\right)+\lambda\left(e_{h}, \alpha_{h}\right) \\
\leq & C h\left\|e_{h}\right\|_{1}\|\varphi\|_{0}+\nu\left(\nabla e_{h}, \nabla \alpha_{h}\right)+\left((u \cdot \nabla) e_{h}, \alpha_{h}\right) \\
& +\left(\left(e_{h} \cdot \nabla\right) u, \alpha_{h}\right)+\lambda\left(e_{h}, \alpha_{h}\right) .
\end{aligned}
$$

For the other terms we take $\phi_{h}=\alpha_{h}$ in (35) and apply (4) to get

$$
\begin{aligned}
& \nu\left(\nabla e_{h}, \nabla \alpha_{h}\right)+\left((u \cdot \nabla) e_{h}, \alpha_{h}\right)+\left(\left(e_{h} \cdot \nabla\right) u, \alpha_{h}\right)+\lambda\left(e_{h}, \alpha_{h}\right) \\
& \quad=\left(\left(\left(w_{h}-v\right) \cdot \nabla\right) u, \alpha_{h}\right)+\lambda\left(w_{h}-v, \alpha_{h}\right) \\
& \quad \leq C\|\nabla u\|_{L^{2 d}}\left\|w_{h}-v\right\|_{0}\left\|\alpha_{h}\right\|_{L^{2 d /(d-1)}}+\lambda\left\|w_{h}-v\right\|_{0}\left\|\alpha_{h}\right\|_{0} \\
& \quad \leq C\|u\|_{2}\left\|w_{h}-v\right\|_{0}\left\|\alpha_{h}\right\|_{1}+\lambda\left\|w_{h}-v\right\|_{0}\left\|\alpha_{h}\right\|_{1} \\
& \quad \leq C\left\|w_{h}-v\right\|_{0}\|\varphi\|_{0},
\end{aligned}
$$

where the constant $C$ depends on $M_{2}$ (see (7)) and the last inequality is due to

$$
\left\|\alpha_{h}\right\|_{1} \leq\left\|\alpha_{h}-\alpha\right\|_{1}+\|\alpha\|_{1} \leq C h\|\alpha\|_{2}+\|\alpha\|_{2} \leq C\|\varphi\|_{0} .
$$

Inserting (44) in (43) and applying (19) we obtain the following bound

$$
\begin{aligned}
\left\|e_{h}\right\|_{0} & \leq C h\left\|e_{h}\right\|_{1}+C\left\|w_{h}-v\right\|_{0} \\
& \leq C h^{m}\left(\|v\|_{m}+\|j\|_{H^{m-1} / \mathbb{R}}\right) .
\end{aligned}
$$

Applying triangle inequality and (19) again we conclude the proof.

\section{Some auxiliary results}

We now state some results that will be used to get the rate of convergence of the new two-grid method. Let us denote by $B_{H}$ the bilinear form defined by

$B_{H}(v, w)=\nu(\nabla v, \nabla w)+\left(\left(u_{H} \cdot \nabla\right) v, w\right)+\left((v \cdot \nabla) u_{H}, w\right)+\lambda(v, w), \quad v, w \in H_{0}^{1}$,

where $u_{H}$ is the mixed finite-element approximation to $u$ defined in (11)-(12). Continuity of $B_{H}$ can be proved in the following way

$B_{H}(v, w)=B_{u}(v, w)-\left(B_{u}-B_{H}\right)(v, w) \leq C\left(\nu+2\|u\|_{1}+2\left\|u_{H}-u\right\|_{1}+\lambda\right)\|v\|_{1}\|w\|_{1}$.

Moreover, for $H$ small enough $B_{H}$, is also coercive. Using the same decomposition as before and the coercivity of the bilinear form $B_{u}$ we get

$$
\begin{aligned}
B_{H}(v, v)=B_{u}(v, v)-\left(B_{u}-B_{H}\right)(v, v) & \geq B_{u}(v, v)-2\left\|u_{H}-u\right\|_{1}\|v\|_{1}^{2} \\
& \geq\left(\nu-2 C \frac{H^{r-1}}{t^{(r-2) / 2}}\right)\|v\|_{1}^{2} .
\end{aligned}
$$


Then, the coercivity is attained when $u_{H}$ is a good enough approximation to $u$, i.e., when $H$ is small enough. More precisely:

Remark 2 Let us observe that for $t>0$ and $H<\left(t^{(r-2) / 2} \nu / 2 C\right)^{1 /(r-1)}$ the bilinear form (46) is coercive. Taking for example $H<\left(t^{(r-2) / 2} \nu /(4 C)\right)^{1 /(r-1)}$ we get

$$
\left|B_{H}(v, v)\right| \geq \frac{\nu}{2}\|v\|_{1}^{2}, \quad \forall v \in H_{0}^{1} .
$$

We want to remark that we are not studying the convection dominating regime in which $\nu \rightarrow 0$. Some works concerning this case are for example, [5], [1] where the continuous interior penalty method and the local projection stabilization method for the Navier-Stokes equations are considered and [10] where a plain Galerkin method with grad-div stabilization for the Navier-Stokes equations is analyzed. In all these papers error bounds with constants independent on the viscosity parameter $\nu$ can be found.

Altogether, we can state that the method can be applied whenever condition (26), that assures coercivity of the continuous problem, is satisfied, for any strictly positive time $t>0$, for any strictly positive value of the viscosity parameter $\nu$ and whenever $H$ is small enough, $H<\left(t^{(r-2) / 2} \nu /(4 C)\right)^{1 /(r-1)}$. Under these assumptions the bilinear form $B_{H}$ is coercive which assures the stability of the method.

The following two lemmas establish some bounds for the temporal derivative of the Galerkin error and for the dual norm or the Galerkin error. Their proofs can be found in [14, Lemma 4] for the case $r=2,[11$, Lemma 5.1] for $r=3,4$ and in $[11$, p. 226], respectively.

Lemma 2 Let $(u, p)$ be the solution of (1) and let $u_{H}$ be the mixed finite-element approximation to $u$ defined in (11)-(12). Let $A$ the Stokes operator defined by (7). Then, there exists a positive constant $C$ such that

$$
\begin{aligned}
\left\|u_{t}(t)-\dot{u}_{H}(t)\right\|_{-1} & \leq \frac{C}{t^{(r-1) / 2}} H^{r}|\log (H)|^{r^{\prime}}, t \in(0, T], r=2,3,4, \\
\left\|A^{-1} \Pi\left(u_{t}(t)-\dot{u}_{H}(t)\right)\right\|_{0} & \leq \frac{C}{t^{(r-1) / 2}} H^{r+1}|\log (H)|, t \in(0, T], r=3,4,
\end{aligned}
$$

where $r^{\prime}=2$ when $r=2$ and $r^{\prime}=1$ otherwise.

Lemma 3 Let $(u, p)$ be the solution of (1) and let $u_{H}$ be the mixed finite-element approximation to $u$ defined in (11)-(12). Then, there exists a positive constant $C$ such that

$$
\left\|u(t)-u_{H}(t)\right\|_{-1} \leq \frac{C}{t^{(r-1) / 2}} H^{r+1}|\log (H)|, t \in(0, T], \quad r=3,4 .
$$

\section{The Newton-type two-grid method}

The postprocessing technique we propose is a two-level or two-grid method. In the first level, we choose a coarse mesh of size $H$ and compute the mixed 
finite-element approximation $\left(u_{H}, p_{H}\right)$ to $(u, p)$ defined by (11)-(12). In the second level, the discrete velocity and pressure $\left(u_{H}(t), p_{H}(t)\right)$ are postprocessed by solving the following linear Newton-type problem: find $\left(\tilde{u}_{h}(t), \tilde{p}_{h}(t)\right) \in$ $\left(X_{h, r}, Q_{h, r-1}\right), h<H$, satisfying for all $\phi_{h} \in X_{h, r}$ and $\psi_{h} \in Q_{h, r-1}$

$$
\begin{array}{r}
\nu\left(\nabla \tilde{u}_{h}(t), \nabla \phi_{h}\right)+\left(\left(u_{H}(t) \cdot \nabla\right) \tilde{u}_{h}(t), \phi_{h}\right)+\left(\left(\tilde{u}_{h}(t) \cdot \nabla\right) u_{H}(t), \phi_{h}\right)+\lambda\left(\tilde{u}_{h}(t), \phi_{h}\right) \\
+\left(\nabla \tilde{p}_{h}(t), \phi_{h}\right)=\left(f(t)-\dot{u}_{H}(t), \phi_{h}\right)+\left(\left(u_{H}(t) \cdot \nabla\right) u_{H}(t), \phi_{h}\right)+\lambda\left(u_{H}, \phi_{h}\right)(50) \\
\left(\nabla \cdot \tilde{u}_{h}(t), \psi_{h}\right)=0 .
\end{array}
$$

Equations (50) can also be solved over a higher order mixed finite-element space over the same grid. For simplicity in the exposition we will only consider the case in which we refine the mesh at the postprocessing step.

We now state the main result of the paper that bounds the error of the post-processed approximation.

Theorem 1 Let $(u, p)$ be the solution of (1) and $\left(\tilde{u}_{h}, \tilde{p}_{h}\right)$ be the solution of (50). Then, for $h$ and $H$ small enough the following bounds hold for $t \in(0, T]$, $m=0,1$ :

$$
\begin{aligned}
\left\|u(t)-\tilde{u}_{h}(t)\right\|_{1} & \leq C h+\frac{C}{t^{1 / 2}} H^{2}|\log (H)|^{2}, r=2, \\
\left\|u(t)-\tilde{u}_{h}(t)\right\|_{m} & \leq \frac{C}{t^{(r-2) / 2}} h^{r-m}+\frac{C}{t^{(r-1) / 2}} H^{r+1-m}|\log (H)|, r=3,4 . \\
\left\|p(t)-\tilde{p}_{h}(t)\right\|_{L^{2} / \mathbb{R}} & \leq \frac{C}{t^{(r-2) / 2}} h^{r-1}+\frac{C}{t^{(r-1) / 2}} H^{r}|\log (H)|^{r^{\prime}}, r=2,3,4,
\end{aligned}
$$

where $r^{\prime}=2$ for $r=2$ and $r^{\prime}=1$ otherwise.

Proof Let us consider the auxiliary problem (21) with $g=f-u_{t}+(u \cdot \nabla) u+\lambda u$. Clearly the solution $(u, p)$ of the Navier-Stokes equations (1) is also the unique solution of this problem. We will denote by $\left(v_{h}, j_{h}\right)$ its mixed finite-element approximation. Let us decompose

$$
u-\tilde{u}_{h}=\left(u-v_{h}\right)+\left(v_{h}-\tilde{u}_{h}\right)
$$

and denote by $e_{h}=v_{h}-\tilde{u}_{h}$. To bound the first term we apply Lemma 1 . For the second, we subtract (50) from (29) projecting onto the discrete divergence free space $V_{h, r}$. Then,

$$
\begin{array}{r}
\nu\left(\nabla e_{h}, \nabla \phi_{h}\right)+\left(\left(u_{H} \cdot \nabla\right) e_{h}, \phi_{h}\right)+\left(\left(e_{h} \cdot \nabla\right) u_{H}, \phi_{h}\right)+\lambda\left(e_{h}, \phi_{h}\right)= \\
\left(\dot{u}_{H}-u_{t}, \phi_{h}\right)+\left(\left(\left(u_{H}-u\right) \cdot \nabla\right) v_{h}, \phi_{h}\right)+\left(\left(v_{h} \cdot \nabla\right)\left(u_{H}-u\right), \phi_{h}\right)+\left((u \cdot \nabla) u, \phi_{h}\right) \\
-\left(\left(u_{H} \cdot \nabla\right) u_{H}, \phi_{h}\right)+\lambda\left(u-u_{H}, \phi_{h}\right), \quad \forall \phi_{h} \in V_{h, r} .
\end{array}
$$

Adding and subtracting to the right-hand side the following terms

$$
\left(\left(\left(u_{H}-u\right) \cdot \nabla\right) u, \phi_{h}\right)+\left((u \cdot \nabla)\left(u_{H}-u\right), \phi_{h}\right)
$$


and reordering terms we get

$$
\begin{array}{r}
\nu\left(\nabla e_{h}, \nabla \phi_{h}\right)+\left(\left(u_{H} \cdot \nabla\right) e_{h}, \phi_{h}\right)+\left(\left(e_{h} \cdot \nabla\right) u_{H}, \phi_{h}\right)+\lambda\left(e_{h}, \phi_{h}\right)= \\
\left(\dot{u}_{H}-u_{t}, \phi_{h}\right)+\left(\left(\left(u_{H}-u\right) \cdot \nabla\right)\left(v_{h}-u\right), \phi_{h}\right)+\left(\left(\left(v_{h}-u\right) \cdot \nabla\right)\left(u_{H}-u\right), \phi_{h}\right) \\
+\left(\left(\left(u_{H}-u\right) \cdot \nabla\right)\left(u-u_{H}\right), \phi_{h}\right)+\lambda\left(u-u_{H}, \phi_{h}\right) .
\end{array}
$$

Taking $\phi_{h}=e_{h}$ and applying (46) we get for $H$ small enough

$$
\begin{aligned}
\frac{\nu}{2}\left\|e_{h}\right\|_{1}^{2} \leq & \left|\left(\dot{u}_{H}-u_{t}, e_{h}\right)\right|+\left|\left(\left(\left(u_{H}-u\right) \cdot \nabla\right)\left(v_{h}-u\right), e_{h}\right)\right| \\
& +\left|\left(\left(\left(v_{h}-u\right) \cdot \nabla\right)\left(u_{H}-u\right), e_{h}\right)\right|+\left|\left(\left(\left(u_{H}-u\right) \cdot \nabla\right)\left(u-u_{H}\right), e_{h}\right)\right| \\
& +\left|\lambda\left(u-u_{H}, e_{h}\right)\right| .
\end{aligned}
$$

We will bound each term on the right-hand side of (55). To this end, we write

$$
\begin{aligned}
\left|\left(\dot{u}_{H}-u_{t}, e_{h}\right)\right| & \leq\left\|\dot{u}_{H}-u_{t}\right\|_{-1}\left\|e_{h}\right\|_{1}, \\
\left|\left(\left(\left(u_{H}-u\right) \cdot \nabla\right)\left(v_{h}-u\right), e_{h}\right)\right| & \leq C\left\|u_{H}-u\right\|_{1}\left\|v_{h}-u\right\|_{1}\left\|e_{h}\right\|_{1}, \\
\left|\left(\left(\left(v_{h}-u\right) \cdot \nabla\right)\left(u_{H}-u\right), e_{h}\right)\right| & \leq C\left\|v_{h}-u\right\|_{1}\left\|u_{H}-u\right\|_{1}\left\|e_{h}\right\|_{1}, \\
\left|\left(\left(\left(u_{H}-u\right) \cdot \nabla\right)\left(u-u_{H}\right), e_{h}\right)\right| & \leq C\left\|u-u_{H}\right\|_{1}^{2}\left\|e_{h}\right\|_{1}, \\
\left|\lambda\left(u-u_{H}, e_{h}\right)\right| & \leq \lambda\left\|u-u_{H}\right\|_{0}\left\|e_{h}\right\|_{0} \leq \lambda\left\|u-u_{H}\right\|_{0}\left\|e_{h}\right\|_{1} .
\end{aligned}
$$

And then we get

$$
\begin{aligned}
\frac{\nu}{2}\left\|e_{h}\right\|_{1} \leq & \left\|u_{t}-\dot{u}_{H}\right\|_{-1}+2 C\left\|v_{h}-u\right\|_{1}\left\|u_{H}-u\right\|_{1} \\
& +C\left\|u-u_{H}\right\|_{1}^{2}+\lambda\left\|u-u_{H}\right\|_{0} .
\end{aligned}
$$

To bound the first term above we apply (47)

$$
\left\|u_{t}-\dot{u}_{H}\right\|_{-1} \leq \frac{C}{t^{(r-1) / 2}} H^{r}|\log (H)|^{r^{\prime}},
$$

where $r^{\prime}=2$ for $r=2$ and $r^{\prime}=1$ for $r=3,4$. For the other terms, we apply (30) with $m=2$ together with (13)

$$
\begin{aligned}
\left\|u-v_{h}\right\|_{1}\left\|u_{H}-u\right\|_{1} & \leq \frac{C}{t^{(r-2) / 2}} h H^{r-1} \\
\left\|u-u_{H}\right\|_{1}^{2} & \leq C \frac{H^{r-1}}{t^{(r-2) / 2}} \frac{H^{\min (2, r-1)}}{t^{1 / 2}}=C \frac{H^{r+\min (1, r-2)}}{t^{(r-1) / 2}}, \\
\left\|u-u_{H}\right\|_{0} & \leq \frac{C}{t^{(r-2) / 2}} H^{r} .
\end{aligned}
$$

Inserting now (57) and (58) in (56) and keeping only the biggest terms in the error bound we get

$$
\left\|e_{h}\right\|_{1} \leq \frac{C}{t^{(r-1) / 2}} H^{r}|\log (H)|^{r^{\prime}}, r=2,3,4,
$$


where $r^{\prime}=2$ for $r=2$ and $r^{\prime}=1$ for $r=3$, 4. Applying triangle inequality together with (30) we conclude

$$
\left\|u(t)-\tilde{u}_{h}(t)\right\|_{1} \leq \frac{C}{t^{(r-2) / 2}} h^{r-1}+\frac{C}{t^{(r-1) / 2}} H^{r}|\log (H)|^{r^{\prime}} .
$$

For the pressure bound, we decompose

$$
p-\tilde{p}_{h}=\left(p-j_{h}\right)+\left(j_{h}-\tilde{p}_{h}\right) .
$$

Let us denote by $r_{h}=j_{h}-\tilde{p}_{h}$. We subtract again (50) from (29) to get

$$
\begin{array}{r}
\nu\left(\nabla e_{h}, \nabla \phi_{h}\right)+\left(\left(u_{H} \cdot \nabla\right) e_{h}, \phi_{h}\right)+\left(\left(e_{h} \cdot \nabla\right) u_{H}, \phi_{h}\right)+\lambda\left(e_{h}, \phi_{h}\right)+\left(\nabla r_{h}, \phi_{h}\right)= \\
\left(\dot{u}_{H}-u_{t}, \phi_{h}\right)+\left(\left(\left(u_{H}-u\right) \cdot \nabla\right)\left(v_{h}-u\right), \phi_{h}\right)+\left(\left(\left(v_{h}-u\right) \cdot \nabla\right)\left(u_{H}-u\right), \phi_{h}\right) \\
+\left(\left(\left(u_{H}-u\right) \cdot \nabla\right)\left(u-u_{H}\right), \phi_{h}\right)+\lambda\left(u-u_{H}, \phi_{h}\right),
\end{array}
$$

for all $\phi_{h} \in X_{h, r}$. Using the continuity of $B_{H}$ and applying the inf-sup condition (10) we get

$$
\begin{aligned}
\beta\left\|r_{h}\right\|_{L^{2} /(R)} \leq & C\left\|e_{h}\right\|_{1}+\left\|u_{t}-\dot{u}_{H}\right\|_{-1}+2 C\left\|v_{h}-u\right\|_{1}\left\|u_{H}-u\right\|_{1} \\
& +C\left\|u-u_{H}\right\|_{1}^{2}+\lambda\left\|u-u_{H}\right\|_{0} .
\end{aligned}
$$

Applying (59) to bound $\left\|e_{h}\right\|_{1}$ together with (57) and (58) we reach

$$
\beta\left\|r_{h}\right\|_{L^{2} /(R)} \leq \frac{C}{t^{(r-1) / 2}} H^{r}|\log (H)|^{r^{\prime}} .
$$

To conclude the error bound for the pressure we apply decomposition (60) and (31).

To bound the $L^{2}$ norm of the error in the velocity we argue by duality exactly as in the proof of Lemma 1 . We write

$$
\left\|e_{h}\right\|_{0}=\sup _{\varphi \in L^{2}(\Omega)^{d}} \frac{\left|\left(e_{h}, \varphi\right)\right|}{\|\varphi\|_{0}},
$$

and consider the following dual problem for each $\varphi \in L^{2}(\Omega)^{d}$

$$
\begin{aligned}
-\nu \Delta \alpha-(u \cdot \nabla) \alpha-\nabla \bar{\alpha} \cdot u+\nabla \gamma+\lambda \alpha= & \varphi, \\
\operatorname{div}(\alpha)= & 0, \\
\alpha=0, & \text { on } \partial \Omega .
\end{aligned}
$$

As in Lemma 1 we denote by $\left(\alpha_{h}, \gamma_{h}\right)$ the Stokes projection of this dual problem. Then, we observe that (43) holds. Using (55) instead of (35) in (43) we reach

$$
\begin{aligned}
\left(e_{h}, \varphi\right) \leq \quad & C h\left\|e_{h}\right\|_{1}\|\varphi\|_{0}+\nu\left(\nabla e_{h}, \nabla \alpha_{h}\right)+\left((u \cdot \nabla) e_{h}, \alpha_{h}\right)+\left(\left(e_{h} \cdot \nabla\right) u, \alpha_{h}\right) \\
& +\lambda\left(e_{h}, \alpha_{h}\right) \\
\leq \quad & C h\left\|e_{h}\right\|_{1}\|\varphi\|_{0}+\left(\dot{u}_{H}-u_{t}, \alpha_{h}\right)+\left(\left(\left(u_{H}-u\right) \cdot \nabla\right)\left(\tilde{u}_{h}-u\right), \alpha_{h}\right) \\
& +\left(\left(\left(\tilde{u}_{h}-u\right) \cdot \nabla\right)\left(u_{H}-u\right), \alpha_{h}\right) \\
& +\left(\left(\left(u_{H}-u\right) \cdot \nabla\right)\left(u-u_{H}\right), \alpha_{h}\right)+\lambda\left(u-u_{H}, \alpha_{h}\right)
\end{aligned}
$$


To get the error bound in $L^{2}$ it is necessary to improve some of the bounds used in the proof of the $H^{1}$ norm. For the temporal Galerkin error, we apply (47), (48) and (41) to obtain

$$
\begin{aligned}
\left|\left(\dot{u}_{H}-u_{t}, \alpha_{h}\right)\right| & =\left|\left(\dot{u}_{H}-u_{t}, \alpha_{h}-\alpha\right)+\left(\dot{u}_{H}-u_{t}, \alpha\right)\right| \\
& \leq C\left\|u_{t}-\dot{u}_{H}\right\|_{-1}\left\|\alpha_{h}-\alpha\right\|_{1}+C\left\|A^{-1} \Pi\left(\dot{u}-u_{t}\right)\right\|_{0}\|A \alpha\|_{0} \\
& \leq C\left\|u_{t}-\dot{u}_{H}\right\|_{-1} h\|\varphi\|_{0}+C\left\|A^{-1} \Pi\left(\dot{u}-u_{t}\right)\right\|_{0}\|\varphi\|_{0} \\
& \leq \frac{C}{t^{(r-1) / 2}} H^{r+1}|\log (H)|\|\varphi\|_{0} .
\end{aligned}
$$

Let us observe that (62) is valid for $r \geq 3$. Next two terms in (61) are bounded applying (52), (13) with $m=2$ and (45)

$$
\begin{aligned}
& \left|\left(\left(\left(u_{H}-u\right) \cdot \nabla\right)\left(\tilde{u}_{h}-u\right), \alpha_{h}\right)\right|+\left|\left(\left(\left(u_{H}-u\right) \cdot \nabla\right)\left(u-u_{H}\right), \alpha_{h}\right)\right| \\
& \quad \leq C\left\|u_{H}-u\right\|_{1}\left\|\tilde{u}_{h}-u\right\|_{1}\left\|\alpha_{h}\right\|_{1} \\
& \quad \leq \frac{C H^{2}}{t^{(r-1) / 2}}\left(t^{1 / 2} h^{r-1}+H^{r}|\log (H)|\right)\|\varphi\|_{0} .
\end{aligned}
$$

For the fifth term we apply (58) and (45) to get

$$
\left|\left(\left(\left(u_{H}-u\right) \cdot \nabla\right)\left(u-u_{H}\right), \alpha_{h}\right)\right| \leq C \frac{H^{r+1}}{t^{(r-1) / 2}}\left\|\varphi_{h}\right\|_{0} .
$$

Finally, for the last term in (62) we use (49) and (45) to obtain

$$
\left|\left(u-u_{H}, \alpha_{h}\right)\right| \leq \frac{C}{t^{(r-1) / 2}} H^{r+1}|\log (H)|\left\|\varphi_{h}\right\|_{0} .
$$

Inserting (62), (63), (64) and (65) in (61) and keeping only the biggest terms in the error bound we get

$$
\left\|e_{h}\right\|_{0} \leq C h\left\|e_{h}\right\|_{1}+\frac{C}{t^{(r-1) / 2}} H^{r+1}|\log (H)| .
$$

Applying (59), decomposition (54) and (30) we finally reach (52) for $m=0$.

Remark 3 We want to observe that projecting onto the discrete divergence free space $V_{h, r}$ in the proof of Theorem 1 simplifies the task of getting the error bounds for the velocity. This technique can be applied whenever inf-sup stable mixed finite elements are considered.

\section{Numerical experiments}

In this section, we carry out several experiments for studying both the order of convergence and the comparative behaviour of the three postprocessed procedures: Stokes, Oseen and Newton. The experiments are computed in the unit square $\Omega=[0,1] \times[0,1]$, using the mini-element over a regular triangulation based on the set of nodes $(i / N, j / N) i, j=1,2 \ldots N$, where $N=1 / H$ is 
the Galerkin spatial resolution. In the time integration we use a semi-implicit trapezoidal rule, where spatial derivatives are treated implicitly. The size of the time step is chosen so that temporal errors are negligible compared to spatial errors. Once the mini-element is obtained, the bubble part is removed and the errors of the linear part are computed. It has been reported in [15] that the linear part is a better approximation to the solution than the complete linearbubble vector velocity. The bubble part is considered only for stability reasons. In this experiment, we take the following functions

$$
\begin{aligned}
u^{1}(x, y, t) & =\pi t \sin ^{2}(\pi x) \sin (2 \pi y), \\
u^{2}(x, y, t) & =-\pi t \sin ^{2}(\pi y) \sin (2 \pi x), \\
p(x, y, t) & =5 t x^{2} y .
\end{aligned}
$$

and calculate the forcing term $f(x, t)$ so that $(66)$ is the solution of the Navier Stokes equations for different values of the diffusion parameter ranging from $\nu=1$ to $\nu=0.001$. The Galerkin approximations are obtained integrating up to time $T=1$ in the coarse mesh of size $H=1 / N$. Then, there are postprocessed at the fixed final time over a finer mesh of size $h<H$ small enough to retain the asymptotic behavior of the rate of convergence. Computational cost of the post-processed step is typically of the order of a single time step over the fine mesh $h$. In this experiment, the sizes of the coarse meshes are given by $N=40, N=50, N=70, N=85$ and for the fine meshes $n=175, n=223$, $n=273$ and $n=353$, respectively. Although for the mini-element, optimal values for the fine mesh in the sense of Theorem 1 are those obtained taking $h=H^{2}$, in practice the rate of convergence of the methods can be reached taking exponents less than 2 . This is the reason why we chose the above values of $n$ that correspond to the smallest values for which increasing them we do not achieve smaller errors.

Figure 1 shows the $L^{2}$ velocity errors of the first component of the velocity for the Galerkin approximation and the three post-processed procedures: Stokes, Oseen and Newton, with respect to the above mentioned values for the coarse and fine meshes. Each of the pictures correspond to a different value of the diffusion parameter, ranging from $\nu=1, \nu=0.1, \nu=0.01$ to $\nu=0.001$ from left to right and up to down. For the Newton-type algorithm we have carried out computations with different values of the parameter $\lambda$. In this experiment, for all values of $\lambda$ we chose in our computations, including $\lambda=0$, the approximations were always computable, i.e., problem (50) had a unique solution. Then, although, theoretically, $\lambda$ should be chosen greater or equal to $\|\nabla u\|_{\infty}$ according to (26) (or in practice $\lambda \geq\left\|\nabla u_{H}\right\| \infty$ since $u$ is in general unknown) in the experiments shown in this section we have chosen the values of $\lambda$ that produce better errors for the method. These values depend on the diffusion parameter. In all the pictures, we have chosen $\lambda=0.1,0.5,1.2$ and 2 for $\nu=1,0.1,0.01$ and 0.001 respectively.

It can be observed in Figure 1 that, as expected, in general the post-processed procedures do not increase the $L^{2}$ rate of convergence of the Galerkin method, although there is a slight increase for $\nu=1$. This is a particular case due to 

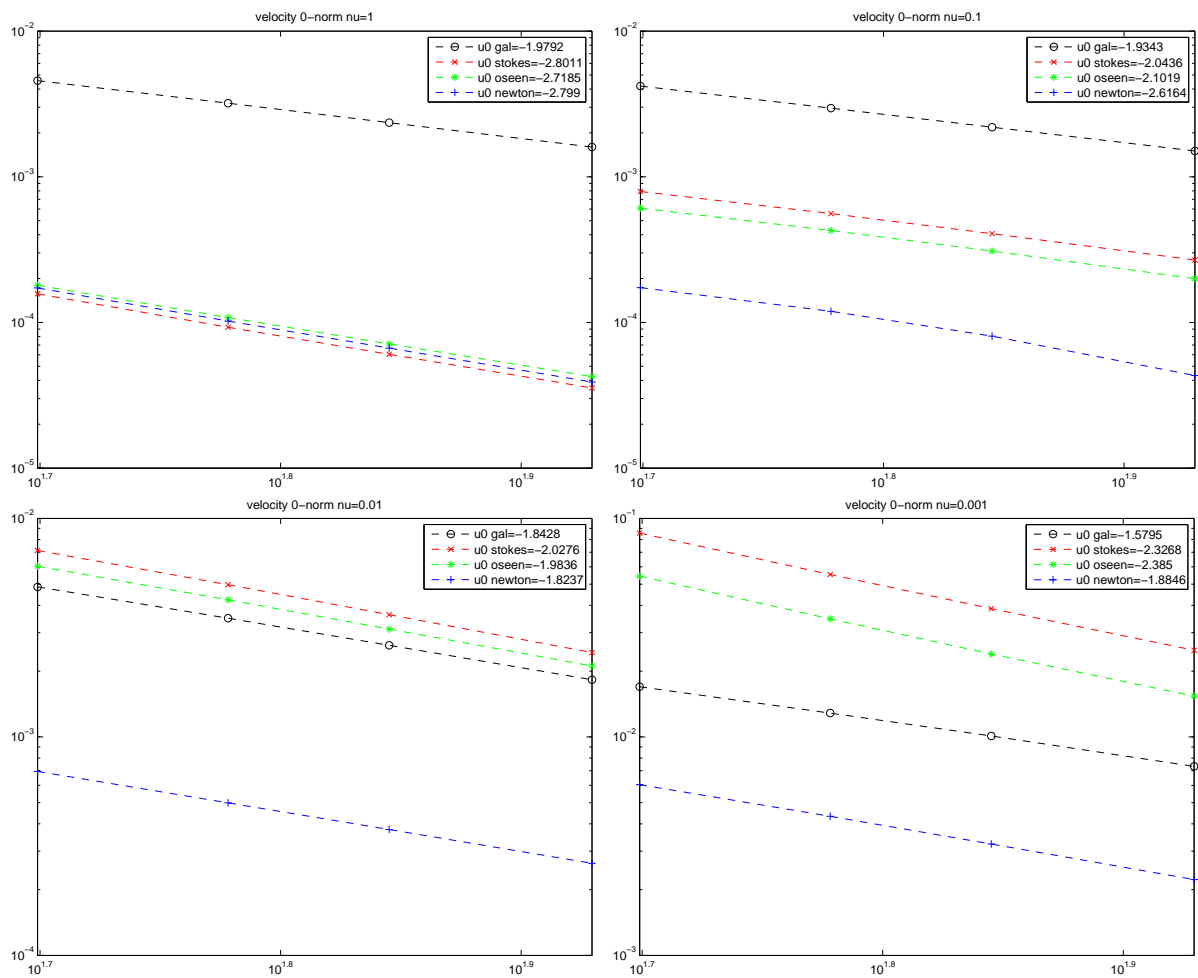

Figure 1: $L^{2}$ errors for the first velocity component with $\nu=1, \nu=0.1, \nu=0.01$ and $\nu=0.001$

the use of linear elements (case $r=2$ ). For $\nu=1$ all the postprocessed methods produce the same errors and much smaller than the Galerkin errors. The comparison between the methods changes with the value of $\nu$. As $\nu$ decreases the difference between the errors of the new method and the other two postprocessed methods increases being for $\nu=0.001$ the new procedure the only one producing much smaller errors than the Galerkin method. As expected, all the methods deteriorate when decreasing the value of $\nu$ due to the loss of coercivity but the new method is the one behaving better.

Figure 2 shows the $H^{1}$ norm of the error for the first component of the velocity. Considering this norm, and in agreement with the theory, it is remarkable the increment of the rate of convergence of the new postprocessed method in one unit in terms of $H$ (same increment can be observed for the other two postprocessed methods). Contrary to the situation shown in Figure 1 for the $L^{2}$ errors, in the $H^{1}$ norm the three postprocessed methods produce always smaller errors than the Galerkin method, being again Newton-type the best post-processing procedure. The Stokes method reveals itself to be the worst postprocess in all cases. Since the Galerkin errors lie on a perfect straight line we can deduce from the figure the Galerkin errors for other values of $N$ different 

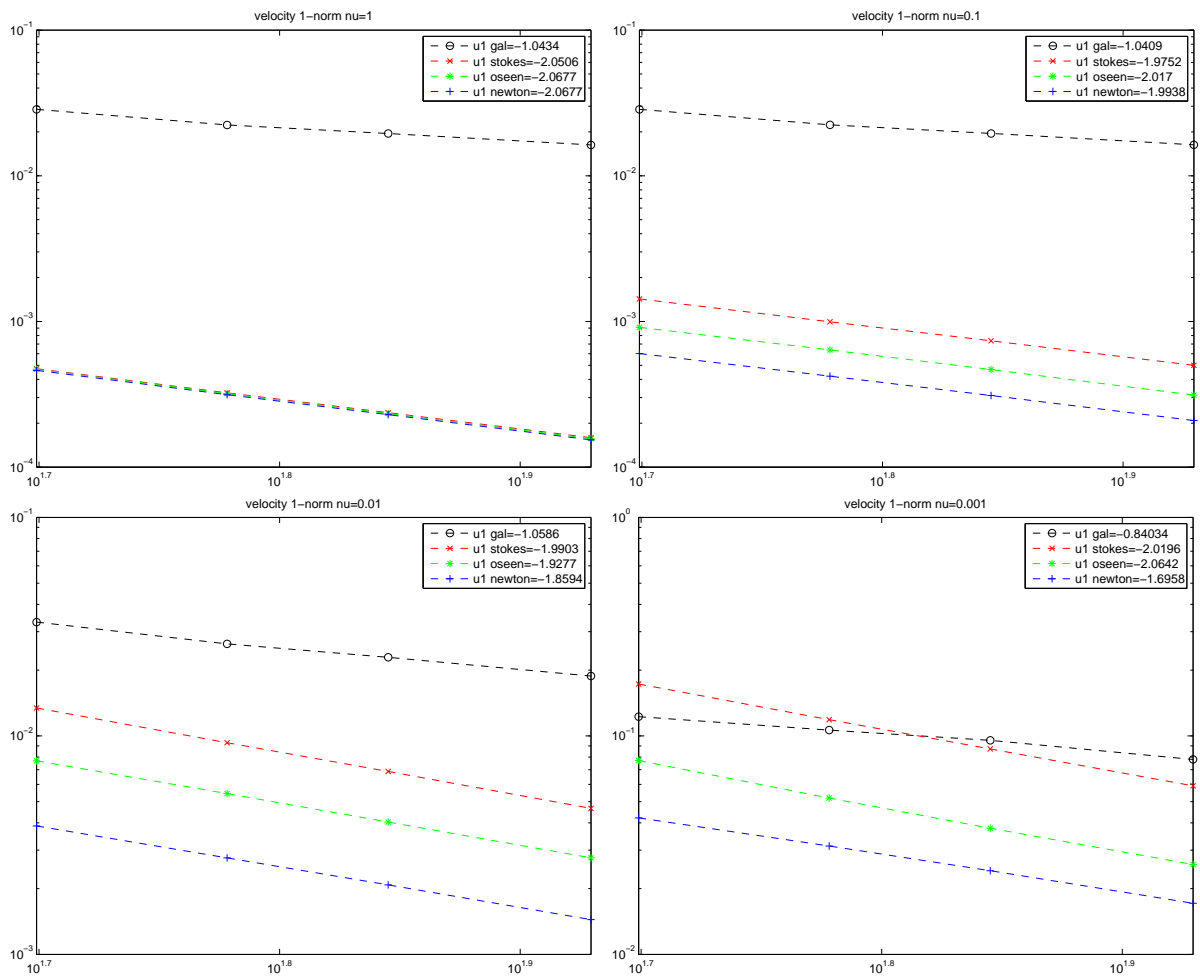

Figure 2: $H^{1}$ errors for the first velocity component with $\nu=1, \nu=0.1$, $\nu=0.01$ and $\nu=0.001$

from those plotted. In particular, for example, for $\nu=0.01$ the Galerkin error for $n=175$ is around 0.008 while the error for $n=353$ is 0.004 . On the other hand, the Newton-type postprocess gives for $(N, n)=(40,175)$ an error of size 0.004 while for the last plotted value $(N, n)=(85,353)$ the error is around 0.001 . This means that although in the picture we have compared the postprocessed errors with the coarse-mesh Galerkin errors if we compare the postprocessed errors with the fine-mesh Galerkin errors the postprocessed procedure gives still better errors than the Galerkin method. However, the computational cost of the postprocessed method is essentially the same as the Galerkin method over the coarse mesh. This means that those errors on the same vertical line are achieved with almost the same computational CPU time. Then, from the picture we can deduce that not only the new method produces smaller errors but is also considerably more efficient.

Finally, Figure 3 shows the $L^{2}$ errors for the pressure. We can observe that the postprocessed pressures of the Newton-type method have a rate of convergence of order 2, according to Theorem 1. As in Figure 1, Newton-type postprocess is the best one, Stokes is never worse than Galerkin while Oseen is worse than the Galerkin method for $\nu=0.001$. 

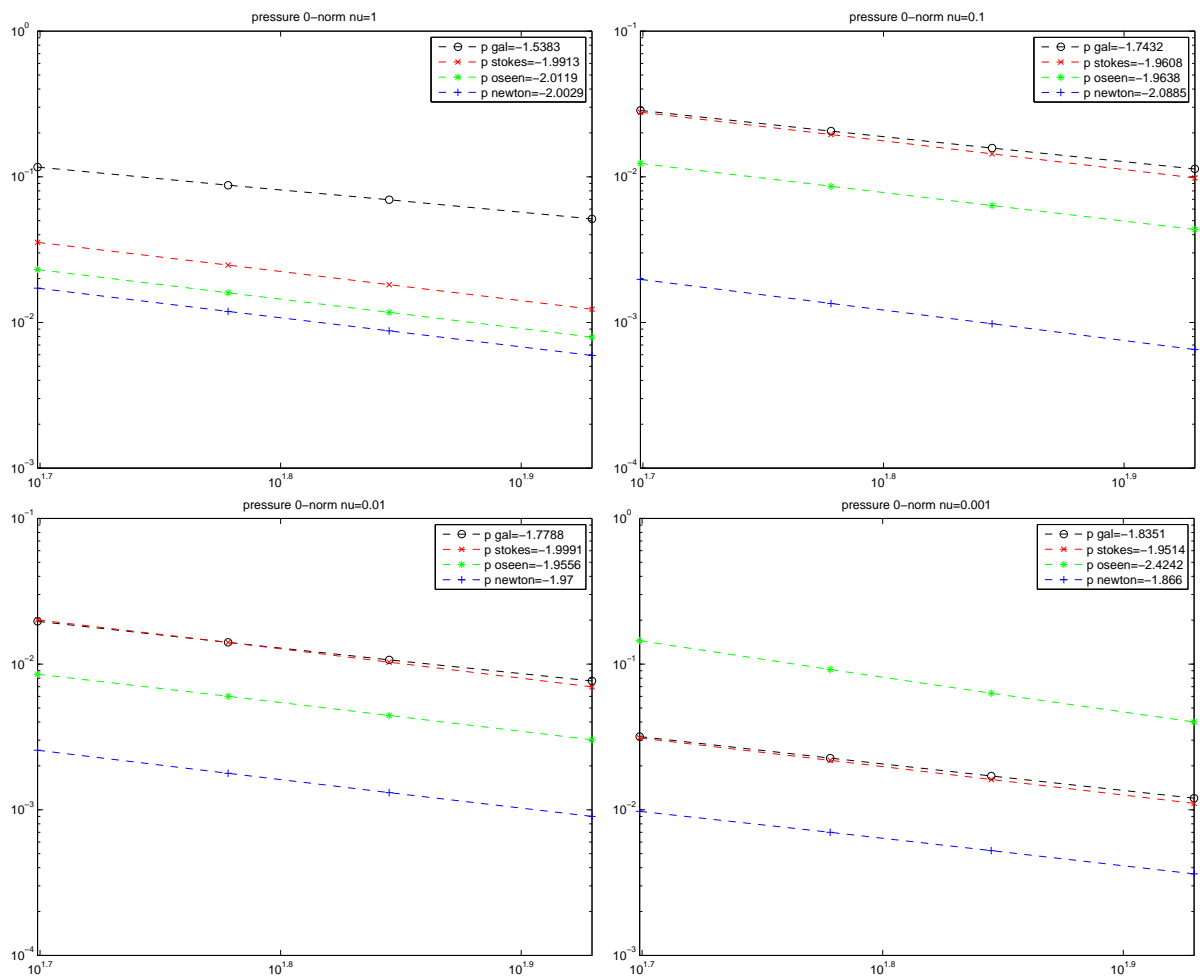

Figure 3: $L^{2}$ errors for the pressure with $\nu=1, \nu=0.1, \nu=0.01$ and $\nu=0.001$

In summary, the new method we analyze is the one giving better errors for all values of the Reynolds number computed in the experiment. Our results are in agreement with the numerical examples of [25] where, as mentioned in the introduction, the three two-grid methods are compared but with a dynamical (time-dependent) implementation of the linearized problem over the fine mesh. We want to remark that the static two-grid approach we analyze has the advantage of being computationally more efficient since a single steady problem is solved at the selected time in which we need to get an approximation over a finer mesh.

Finally, we want to mention that the increased accuracy of the new Newtontype postprocessing compared with the plain Galerkin method can be applied for the interesting task of getting a posteriori error estimations for the Galerkin method as was already studied in [14] for the Stokes postprocessing. We want to explore the behavior of a similar a posteriori error estimation but based on the Newton-type postprocessing in a future work. 


\section{References}

[1] D. Arndt, H. Dallmann and G. Lube, Local projection FEM stabilization for the time-dependent incompressible Navier-Stokes problem., Numer. Methods Partial Diferential Equations, 31(4) (2015), pp. 1224-1250.

[2] B. Ayuso, J. de Frutos and J. Novo, Improving the accuracy of the mini-element approximation to Navier-Stokes equations, IMA J. Numer. Anal., 27 (2007), pp. 198-218.

[3] B. Ayuso, B. Garca-Archilla and J. Novo, The postprocessed mixed finite element method for the Navier-Stokes equations, SIAM J. Numer. Anal., 43 (2005), pp. 1091-1111.

[4] F. BREzzI And R. S. FALK,Stability of higher-order Hood-Taylor methods, SIAM J. Numer. Anal., 28 (1991), pp. 581-590.

[5] E. Burman and M. A. Fernández, Continuous interior penalty finite element method for the time-dependent Navier-Stokes equations: space discretization and convergence., Numer. Math., 107(1) (2007), pp. 39-77.

[6] B. García-Archilla, J. Novo, and E. S. Titi, Postprocessing the Galerkin method: A novel approach to approximate inertial manifolds, SIAM J. Numer. Anal., 35 (1998), pp. 941-972.

[7] B. García-Archilla, J. Novo, and E. S. Titi, An approximate inertial manifold approach to postprocessing Galerkin methods for the NavierStokes equations, Math. Comp., 68 (1999), pp. 893-911.

[8] F. Brezzi And M. Fortin, Mixed and Hybrid Finite Element Methods, Springer, New York, 1991.

[9] H. Dallmann And D. Arndt, Stabilized finite element methods for the Oberbeck-Boussinesq model. J. Sci. Comput. 69 (2016), pp. 244273.

[10] J. De Frutos, B. García-Archilla, V. John and J. Novo, Analysis of the grad-div stabilization for the time-dependent Navier-Stokes equations with inf-sup stable finite elements, sumitted.

[11] J. De Frutos, B. García-Archilla and J. Novo, The postprocessed mixed finite-element method for the Navier-Stokes equations: refined error bounds, SIAM J. Numer. Anal., 46 (2007), pp. 201-230.

[12] J. De Frutos, B. García-Archilla and J. Novo, Postprocessing Finite-Element Methods for the Navier-Stokes Equations: The Fully Discrete Case, SIAM J. Numer. Anal., 47 (2008), pp. 596-621.

[13] J. De Frutos And J. Novo, A spectral element method for the NavierStokes equations with improved accuracy, SIAM J. Numer. Anal., 38 (2000), pp. 799-819. 
[14] J. de Frutos, B. García-Archilla and J. Novo, A posteriori error estimations for mixed finite-element approximations to the Navier-Stokes equations, J. Comput. Appl. Math. (2011), doi: 10.1016/j.cam.2011.07.033.

[15] J. De Frutos, B. García-Archilla \& J. Novo, Static two-grid mixed finite element approximations to the Navier-Stokes equations, J. Sci. Comput., 52 (2012) 619-637.

[16] J. De Frutos, B. García-Archilla \& J. Novo, Optimal error bounds for two-grid schemes applied to the Navier-Stokes equations, Applied Mathematics and Computation, 218 (2012) 7034-7051.

[17] D. Gilbarg, N. S. Trudinger, Elliptic partial differential equations of second order, Springer, Berlin, 2001.

[18] V. Girault, P.A. Raviart, Finite element methods for the NavierStokes equations. Theory and algorithms, Springer Series Comput. Math. 5, Springer-Verlag Berlin, 1986.

[19] Goswami, D., Damázio, P. D. A two-grid finite element method for timedependent incompressible Navier-Stokes equations with non-smooth initial data. Numer. Math. Theory Methods Appl. 8 (2015), 549-581.

[20] J. G. Heywood and R. Rannacher, Finite element approximation of the nonstationary Navier-Stokes problem. I. Regularity of solutions and second-order error estimates for spatial discretization, SIAM J. Numer. Anal., 19 (1982), pp. 275-311.

[21] J. G. HeYwood And R. Rannacher, Finite element approximation of the nonstationary Navier-Stokes problem. III: Smoothing property and higher order error estimates for spatial discretization, SIAM J. Numer. Anal., 25 (1988), pp. 489-512.

[22] P. Hood AND C. TAYlor, A numerical solution of the Navier-Stokes equations using the finite element technique, Comput. Fluids, 1 (1973), pp. $73-100$.

[23] W. Layton and L. Tobiska, A Two-Level method with backtracking for the Navier-Stokes equations, SIAM J. Numer. Anal., 35 (1998), pp. 20352054 .

[24] W. Layton and W. Lenferink, Two-Level Picard and Modified Picard Methods for the Navier-Stokes Equations, Appl. Math. Comput., 80 (1995), pp. 1-12.

[25] Liu, Q., Hou, Y., Wang, W. And ZhaO, J., Two-level consitent splitting methods based on three corrections for the time-dependent Navier-Stokes equations, Inter. J. Numer. Methods Fluids (2015) DOI: 10.1002/fld.4087. 
[26] L. G. Margolin, E. S. Titi and S. Wynne, The postprocessing Galerkin and nonlinear Galerkin methods-A truncation analysis point of view, SIAM J. Numer. Anal., 41 (2003), pp. 695-714. 